Open Access

\title{
A case of mantle cell lymphoma presenting as lgG4-related dacryoadenitis and sialoadenitis, so-called Mikulicz's disease
}

\author{
Yoshikazu Hayashi ${ }^{1}$, Masafumi Moriyama ${ }^{1 *}$, Takashi Maehara', Yuichi Goto ${ }^{1}$, Shintaro Kawano ${ }^{1}$, Miho Ohta', \\ Akihiko Tanaka', Sachiko Furukawa', Jun-Nosuke Hayashida', Tamotsu Kiyoshima², Mayumi Shimizu', \\ Toru Chikui ${ }^{3}$ and Seiji Nakamura ${ }^{1}$
}

\begin{abstract}
Background: Mantle cell lymphoma (MCL) is a relatively uncommon type of non-Hodgkin lymphoma. It develops in the outer edge of a lymph node called the mantle zone. In contrast, IgG4-related dacryoadenitis and sialoadenitis (IgG4-DS) is characterized by elevated serum lgG4 and persistent bilateral enlargement of lacrimal glands (LGs) and salivary glands (SGs), with infiltration of IgG4-positive plasma cells. Recent studies indicated the importance of differentiation between IgG4-DS and malignant lymphoma.

Case presentation: An 82-year-old man was suspected of IgG4-DS because of a high serum lgG level (2174 mg/dL) and bilateral swelling of LGs and SGs. Lip biopsy and fine needle biopsy of submandibular gland were performed, and subsequently, MCL was diagnosed through the histopathological findings.

Conclusions: MCL most commonly occurs in the Waldeyer ring, but rarely in the stomach, spleen, skin, LG, and SG. We report an unusual case of MCL involving LGs and SGs mimicking lgG4-DS, which suggests that IgG4 testing may be useful in the differentiation of IgG4-DS in the presence of bilateral swelling of LGs or SGs.
\end{abstract}

Keywords: Mantle cell lymphoma, IgG4-related dacryoadenitis and sialoadenitis, Lacrimal glands, Salivary glands

\section{Background}

Mantle cell lymphoma (MCL) is a relatively rare type of non-Hodgkin lymphoma, accounting for $\sim 3 \%$ of malignant lymphoma in Japan. MCL typically occurs in middle-aged to older adults, with a marked male predilection, and has a poorer prognosis compared with other subtypes of non-Hodgkin lymphoma [1, 2]. MCL more often presents in stage III/IV with lymphadenopathy, hepatosplenomegaly, bone marrow involvement, and leukemic spread [3]. Although the majority of MCLs occur in lymph nodes, $25 \%$ of patients have extranodal involvement, with the Waldeyer ring $(6.3 \%)$, intestine (5\%), stomach (2.5\%), orbit (2.5\%), and salivary gland (2.5\%) [4]. Definitive diagnosis of MCL is predicated on appropriate immunohistochemical staining with or

\footnotetext{
*Correspondence: moriyama@dent.kyushu-u.ac.jp

${ }^{1}$ Section of Oral and Maxillofacial Oncology, Division of Maxillofacial Diagnostic and Surgical Sciences, Faculty of Dental Science, Kyushu University, 3-1-1 Maidashi, Higashi-ku, Fukuoka 812-8582, Japan Full list of author information is available at the end of the article
}

without ancillary molecular and flow cytometric studies. Clinicopathological characteristics of MCL are characterized by overexpression of cyclin D1 protein; a feature not seen in other similar-appearing lymphomas.

IgG4-related dacryoadenitis and sialoadenitis (IgG4-DS), also known as Mikulicz's disease, is a unique condition characterized by enlargement of the lacrimal glands (LGs) and salivary glands (SGs) caused by infiltration of lymphocytes. IgG4-DS has been considered to be a subtype of Sjögren's syndrome (SS) because of certain histopathological similarities, particularly lymphocytic infiltration [5]. However, IgG4-DS patients show elevated serum levels of IgG4 and infiltrating IgG4-positive plasma cells in the glandular tissues $[6,7]$. Similar findings have also been identified in other diseases such as autoimmune pancreatitis [8], interstitial pneumonia [9], retroperitoneal fibrosis [10], and sclerosing cholangitis [11], and these diseases are now referred to as IgG4-related disease (IgG4-RD) [12]. IgG4-DS is now diagnosed by both 
"Comprehensive Diagnostic Criteria for IgG4-related Disease (2011)" and "Diagnostic Criteria for IgG4related Mikulicz's Disease" approved by the Japanese Society for Sjögren's syndrome [13]. However, it is important to differentiate IgG4-RD from malignant tumors (such as cancer and lymphoma) and similar diseases (such as SS, primary sclerosing cholangitis, Castleman's disease, secondary retroperitoneal fibrosis Wegener's granulomatosis, sarcoidosis, and Churg-Strauss syndrome) by histopathological examination of local lesions. Recently, we reported a case of marginal zone B-cell lymphoma mimicking IgG4-DS [14]. Here, we report a rare case of MCL involving multiple lymph nodes and bilateral LGs and SGs mimicking IgG4-DS.

\section{Case presentation}

An 82-year-old man was referred to our institution with bilateral swelling of the LGs and submandibular glands (SMGs) in November 2008. He had previously attended the ophthalmology department in our hospital with chief complaints of bilateral swelling of LGs, which were diagnosed as IgG4-DS by these clinical findings and high serum IgG (2174 mg/dL). LG biopsy was performed for definitive diagnosis and 2 days later he visited our institution on referral from our ophthalmology department for further evaluation of SMGs. He had several medical histories of colonic cancer, hypertension, and cerebral infarction.
Physical findings showed no fever (body temperature, $36.3{ }^{\circ} \mathrm{C}$ ), blood pressure of $132 / 78 \mathrm{mmHg}$, no weight loss, and no nocturnal sweating. He was aware of dry mouth (unstimulated salivary flow rate, $0.4 \mathrm{~mL} /$ $15 \mathrm{~min}$ ). Bilateral SMGs, sublingual glands (SLGs), and labial salivary glands (LSGs) were elastic, hard and swollen without pain (Fig. 1). Several cervical lymph nodes were enlarged. Computed tomography (CT) showed marked swelling of bilateral LGs and SMGs (Fig. 2a). F-18 fluorodeoxyglucose positron emission tomography demonstrated abnormal multiple accumulations in the LGs (SUVmax, 7.14), SMGs (SUVmax, 6.83), and several systemic lymph nodes (SUVmax, 3.45-4.74), including in the neck and mediastinum (Fig. 2b).

He had a low hemoglobin level $(13.4 \mathrm{~g} / \mathrm{dL})$, red blood cell count of $4.57 \times 10^{6} / \mathrm{mm}^{3}$, white blood cell count of $7150 / \mathrm{mm}^{3}$ (neutrophils $45.5 \%$, lymphocytes $35.0 \%$, monocytes $9.5 \%$, eosinophils $1.0 \%$, and basophils $0.5 \%$, and platelet count of $179 \times 10^{3} / \mathrm{mm}^{3}$. His Creactive protein level was $0.23 \mathrm{mg} / \mathrm{dL}$. The serum soluble interluekin-2 receptor concentration was abnormally high (2251.4 U/mL). Immunological tests were negative for the anti-SS-A/B antibody and the anti-thyroid peroxidase antibody, but positive for rheumatoid factor. Serum levels of IgA, IgM, and IgE were within normal limits (354 $\mathrm{mg} / \mathrm{dL}, 167 \mathrm{mg} / \mathrm{dL}$, and $44 \mathrm{IU} / \mathrm{mL}$, respectively), but his serum IgG level was elevated $(2174 \mathrm{mg} / \mathrm{dL})$.
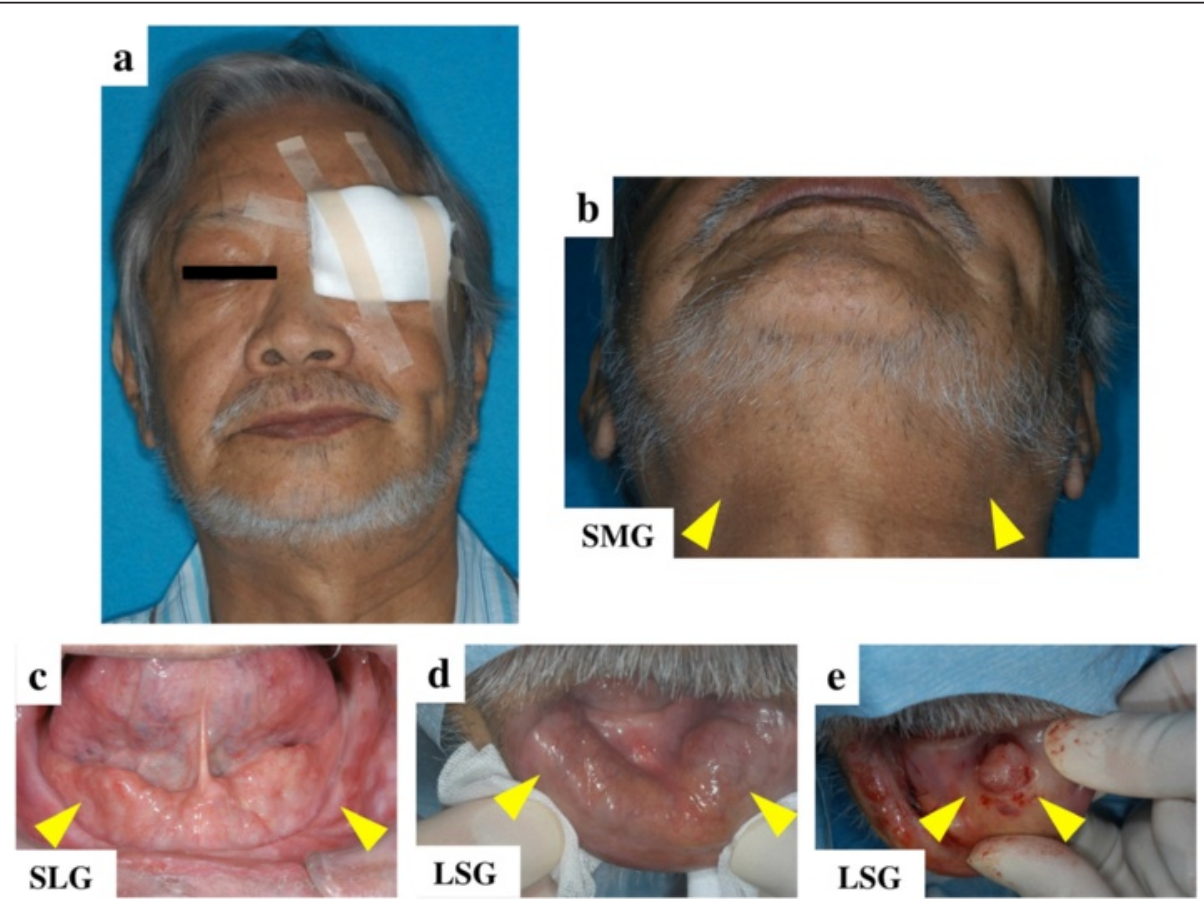

Fig. 1 Clinical findings before treatment. Bilateral swelling of LGs, SMGs, and SLGs (yellow arrowheads) (a-c). Multiple swelling of LSGs at the time of biopsy (blue arrowheads) (d, e) 
a
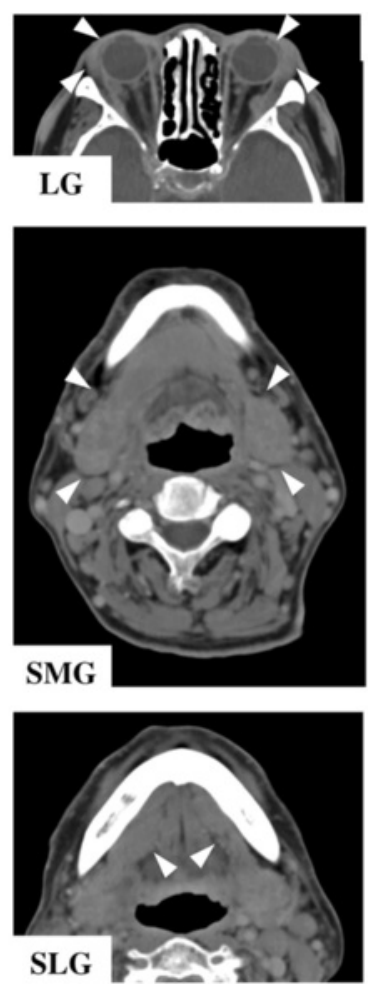

b
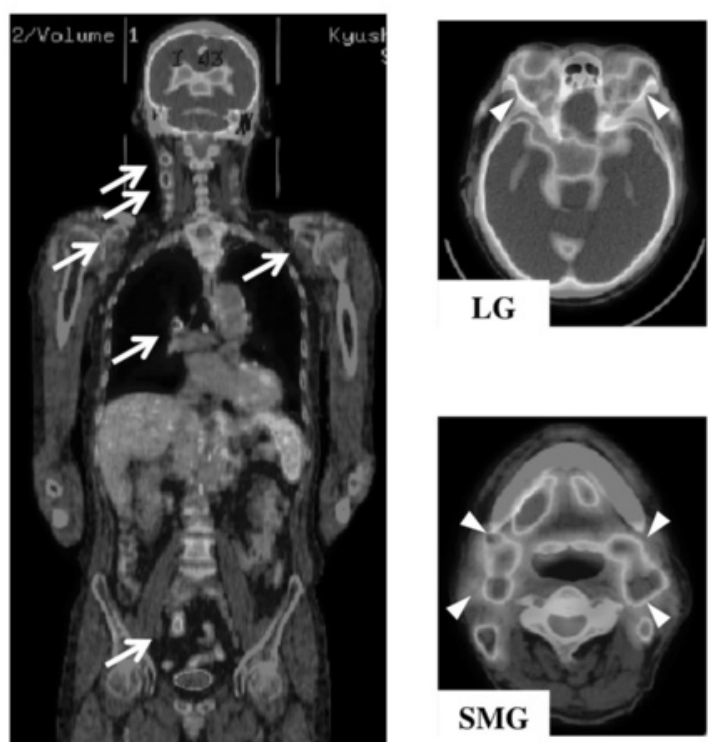

Fig. 2 Imaging findings before treatment. a CT findings indicating swelling of LGs, SMGs and SLGs (white arrowheads). b FDG-PET indicating abnormal multiple accumulations in SMGs and LGs (white arrowheads) and in multiple systemic lymph nodes (white arrows)

IgG4-DS was suspected because of persistent symmetrical swelling of at least two pairs of LGs and major SGs for at least 3 months. We thus additionally examined serum IgG4 and it was in the normal range $(43.7 \mathrm{mg} / \mathrm{dL})$. We performed LSG biopsy and fine needle biopsy (FNB) of the left SMG in addition to LG biospy to check if LSG and SMG were the same disease. Histologically, all sections showed severe uniform infiltration of lymphoplasmacytes, without lymphoid follicular formation. The plasmacytoid cells showed nuclear pleomorphism. Immunohistochemical staining showed monotypic predominance of kappa-light chain and no infiltration of IgG4-positive plasma cells. The infiltrating lymphocytes were positive for B-cell markers (CD20 and CD79a), CD5, bcl-2, and cyclin D1, but negative for T-cell markers (CD3) and CD10 (Fig. 3).

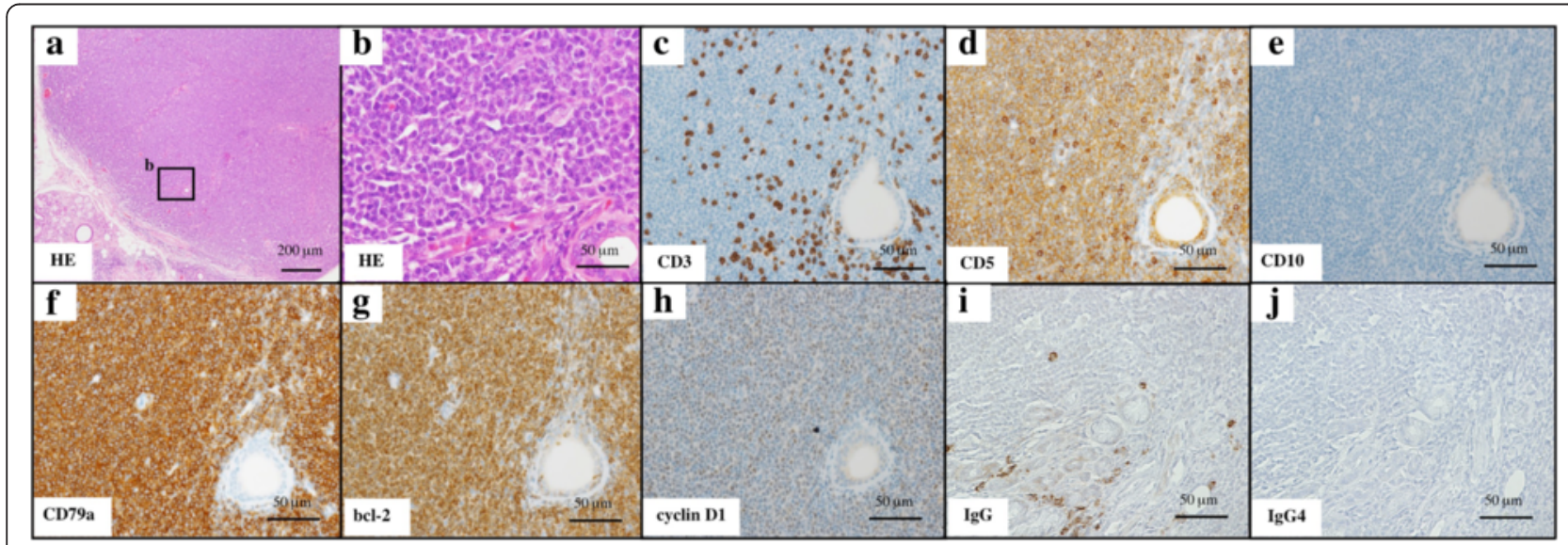

Fig. 3 Histological findings in LSG. Marked lymphoplasmacytic infiltration with hyperplastic lymphoid follicles. The infiltrating lymphocytes were stained with hematoxylin and eosin (HE) (a, b), anti-CD3 (c), anti-CD5 (d), anti-CD10 (e), anti-CD79a (f), anti-bcl-2 (g), anti-cyclin D1 (h), lgG (i), and $\operatorname{lgG} 4$ (j) monoclonal antibodies 
Table 1 Differential diagnosis of lymphoma in immunostaining

\begin{tabular}{|c|c|c|c|c|c|c|c|c|}
\hline & CD3 & CD5 & CD10 & CD20 & CD79a & Cyclin D1 & $\mathrm{bcl}-2$ & UCHL-1 \\
\hline DLBL & - & - & $+/-$ & + & + & - & $+/-$ & - \\
\hline $\mathrm{FL}$ & - & - & + & + & + & - & + & - \\
\hline $\begin{array}{l}\text { MALT } \\
\text { lymphoma }\end{array}$ & - & - & - & + & + & - & + & - \\
\hline$M C L$ & - & + & - & + & + & + & + & - \\
\hline This case & - & + & - & + & + & + & + & - \\
\hline
\end{tabular}

DLBL diffuse large B-cell lymphoma, $F L$ follicular lymphoma, MALT mucosa-associated lymphoid tissue

These histopathological findings and clinical features confirmed a diagnosis of MCL. The patient underwent radiotherapy (20 Gy/10 Fr) and chemotherapy (600 mg rituximab, four times). Although swelling of the LGs and SMGs diminished markedly, it became intractable to treatment at 1 year following therapy and finally terminated in patient death in November 2011.

\section{Conclusions}

MCL is recognized as an aggressive lymphoma for which the median survival of patients is $\sim 3$ years [15]. The clinical and prognostic implications of initial presentation of MCL in the hard palate are currently unknown. MCL is presently staged by using a modified Ann Arbor system [16]. Stage I consists of the involvement of a lymphoid structure or lymph node region, whereas stage II exhibits the involvement of two or more regions on the same side of the diaphragm. Stage III disease represents the involvement of structures or lymph node regions on both sides of the diaphragm, whereas stage IV disease is characterized by diffuse disseminated involvement of one or more extralymphatic organs, such as bone marrow, liver, or lung. Our patient had stage II disease at presentation, with lesions of the LGs, SGs, and cervical lymph nodes.

Immunophenotypically, the neoplastic lymphocytes usually express CD5, CD20, CD43, and FMC-7, but not CD10 or Bcl-6 [17]. The lymphocytes are typically negative for CD23 expression, but on occasion, they may be weakly positive. All lymphocytic specimens typically express Bcl-1, including the rare specimen that is CD5 negative [18]. An important, but less-recognized fact is that almost all lymphocytes are Bcl-2 positive. Several studies have reported that immunohistochemical detection of cyclin D1 is useful for diagnosis of MCL [19-21]. In this case, we confirmed that the patient was positive for CD-5 and cyclin D1 and negative for CD-10. This suggested that this case was consistent with MCL (Table 1).

Larry et al. [4] reported clinical characteristics of primary MCLs. According to their study, 20 of 80 MCLs involved extranodal sites, while only two involved SGs. To the best of our knowledge, two cases of MCL were reported to involve bilateral LGs and parotid glands (Table 2) $[22,23]$. Therefore, the present case report of MCL occurring in bilateral LGs and SMGs is rare, and these clinical findings were similar to those in IgG4-DS. We thus additionally examined serum IgG4 levels and performed both LSG biopsy and FNB of swelling salivary glands.

IgG4-DS is now recognized as a new emerging disorder, characterized by high serum IgG4, marked infiltration of IgG4-positive plasma cells, and severe fibrosis with hyperplastic ectopic germinal centers in LGs and SGs. We recently proposed "Comprehensive Diagnostic Criteria for IgG4-RD" [9]. IgG4-RD can be diagnosed using these criteria combined with organ-specific criteria. If a diagnosis of IgG4-DS is probable or possible based on these criteria, it can be confirmed according to the "Diagnostic Criteria for IgG4-related Mikulicz's Disease" approved by the Japanese Society for Sjögren's Syndrome in 2008, which include the following items: (i) persistent ( $>3$ months) symmetrical swelling of more than two LGs and major SGs; (ii) raised serum levels of IgG4 (>135 mg/dL); and (iii) infiltration of IgG4-positive plasma cells in the tissue (IgG4-positive plasma cells/ IgG-positive plasma cells $>0.4$ ) by immunostaining. For a positive diagnosis of IgG4-DS, any two of these three criteria must be fulfilled, including item (i). The present case met criterion (i), and IgG4-DS was therefore suspected. However, biopsy of the local lesion is recommended for differential diagnosis from other disorders, including sarcoidosis, Castleman's disease, Wegener's granulomatosis, lymphoma, and cancer. We therefore performed LSG biopsy and FNB of SMGs, resulting in a definitive diagnosis of MCL. These results suggest that biopsy of the swollen lesion is essential for a definitive diagnosis of IgG4-DS. Moreover, we recently reported the importance of the utility of SMG incisional biopsies [24]. In conclusion, we emphasize the importance of performing thorough biopsy and serum IgG4 testing

Table 2 MCL involved in LGs and SGS

\begin{tabular}{lllllll}
\hline Case & Sex & Age & Regions & & Ann Arbor staging & Author \\
\hline 1 & Female & 42 & LG, PG, SMG & Bilateral & Palaniswamy [22] & 2009 \\
2 & Male & 52 & LG, PG, SMG & Bilateral & Sagar [21] & 2011 \\
3 & Male & 82 & LG, PG, SMG & Bilateral & IIE & This case
\end{tabular}


when making an accurate diagnosis of bilateral LG and SG swelling. Therefore, we suggest that accurate and rapid diagnosis leads to effective treatment.

\section{Consent}

This study design was approved by the Ethics Committee of Kyushu University, Japan, and written informed consent was obtained from all of the patients and healthy controls (IRB serial number: 25-287).

\section{Abbreviations}

CT: computed tomography; IgG4-DS: IgG4-related dacryoadenitis and sialoadenitis; IgG4-RD: IgG4-related disease; LG: lacrimal gland; LSG: labial salivary gland; MCL: mantle cell lymphoma; MD: Mikulicz's disease; SLG: sublingual gland; SMG: submandibular gland.

\section{Competing interests}

The authors declare that they have no competing interests.

\section{Authors' contributions}

YH and MM participated in study design, literature search, data analysis, manuscript writing, and editing. In addition, TM participated in submission of the manuscript. YG, SK, AT, SF, MO, J-NH, TK, MS, and TC participated in data analysis, manuscript writing, and editing. In addition, SN supervised the study. All the authors read and approved the final manuscript.

\section{Acknowledgements}

This work was supported in part by grants from the Ministry of Education, Culture, Sports, Science, and Technology of Japan (26293430, 26670869), the "Research on Measures for Intractable Diseases" Project, a matching fund subsidy from the Ministry of Health Labour and Welfare, Japan (H26-026, H26-050, H26-064, H26-083) and the "Takeda Science Foundation".

\section{Author details}

${ }^{1}$ Section of Oral and Maxillofacial Oncology, Division of Maxillofacial Diagnostic and Surgical Sciences, Faculty of Dental Science, Kyushu University, 3-1-1 Maidashi, Higashi-ku, Fukuoka 812-8582, Japan. ${ }^{2}$ Laboratory of Oral Pathology, Division of Maxillofacial Diagnostic and Surgical Sciences, Faculty of Dental Science, Kyushu University, 3-1-1 Maidashi, Higashi-ku, Fukuoka 812-8582, Japan. ${ }^{3}$ Department of Oral and Maxillofacial Radiology, Kyushu University Hospital, 3-1-1 Maidashi, Higashi-ku, Fukuoka 812-8582, Japan.

Received: 1 June 2015 Accepted: 8 July 2015

Published online: 25 July 2015

\section{References}

1. Zhou Y, Wang H, Fang W, Romaguer JE, Zhang Y, Delasalle KB, et al. Incidence trends of mantle cell lymphoma in the United States between 1992 and 2004. Cancer. 2008:113:791-8.

2. Norton AJ, Matthews J, Pappa V, Shamash J, Love S, Rohatiner AZ, et al. Mantle cell lymphoma: natural history defined in a serially biopsied population over a 20-year period. Ann Oncol. 1995;6:249-56.

3. Jares $P, C a m p o E$. Advances in the understanding of mantle cell lymphoma. Br J Haematol. 2008;142:149-65.

4. Argatoff $L H$, Connors JM, Klasa RJ, Horsman DE, Gascoyne RD. Mantle cell lymphoma: a clinicopathologic study of 80 cases. Blood. 1997;89:2067-78.

5. Morgan WS, Castleman B. A clinicopathologic study of Mikulicz's disease. Am J Pathol. 1953:29:471-503.

6. Yamamoto M, Takahashi H, Sugai S, Imai K. Clinical and pathological characteristics of Mikulicz's disease (IgG4-related plasmacytic exocrinopathy). Autoimmun Rev. 2005;4:195-200.

7. Yamamoto M, Harada S, Ohara M, Suzuki C, Naishiro Y, Yamamoto H, et al. Clinical and pathological differences between Mikulicz's disease and Sjogren's syndrome. Rheumatology (Oxford). 2005;44:227-34.

8. Hamano H, Kawa S, Horiuchi A, Unno H, Furuya N, Akamatsu T, et al. High serum IgG4 concentrations in patients with sclerosing pancreatitis. N Engl J Med. 2001;344:732-8.
9. Uchiyama-Tanaka Y, Mori Y, Kimura T, Sonomura K, Umemura S, Kishimoto N, et al. Acute tubulointerstitial nephritis associated with autoimmune-related pancreatitis. Am J Kidney Dis. 2004;43:e18-25.

10. Kamisawa T, Funata N, Hayashi Y, Eishi Y, Koike M, Tsuruta K, et al. A new clinicopathological entity of IgG4-related autoimmune disease. J Gastroenterol. 2003;38:982-4.

11. Nakazawa T, Ohara H, Sano H, Aoki S, Kobayashi S, Okamoto T, et al. Cholangiography can discriminate sclerosing cholangitis with autoimmune pancreatitis from primary sclerosing cholangitis. Gastrointest Endosc. 2004;60:937-44.

12. Umehara H, Okazaki K, Masaki Y, Kawano M, Yamamoto M, Saeki T, et al. A novel clinical entity, lgG4-related disease (IgG4RD): general concept and details. Mod Rheumatol. 2012;22:1-14.

13. Umehara H, Okazaki K, Masaki Y, Kawano M, Yamamoto M, Saeki T, et al. Comprehensive diagnostic criteria for lgG4-related disease (IgG4-RD), 2011. Mod Rheumatol. 2012;22:21-30.

14. Ohta M, Moriyama M, Goto Y, Kawano S, Tanaka A, Maehara T, et al. A case of marginal zone B cell lymphoma mimicking IgG4-related dacryoadenitis and sialoadenitis. World J Surg Oncol. 2015;13:67.

15. Harris NL, Jaffe ES, Stein H, Banks PM, Chan JK, Cleary ML, et al. A revised European-American classification of lymphoid neoplasms: a proposal from the International Lymphoma Study Group. Blood. 1994;84:1361-92.

16. Fleming IEA. AJCC cancer staging manual. Philadelphia: Lippincott-Raven; 1997. p. 290.

17. Zucca E, Stein H, Coiffier B. European Lymphoma Task Force (ELTF): report of the workshop on mantle cell lymphoma (MCL). Ann Oncol. 1994;5:507-11.

18. Liu Z, Dong HY, Gorczyca W, Tsang P, Cohen P, Stephenson CF, et al. CD5-mantle cell lymphoma. Am J Clin Pathol. 2002;1 18:216-24.

19. Yang WI, Zukerberg LR, Motokura T, Arnold A, Harris NL. Cyclin D1 (BCl-1, PRAD1) protein expression in low-grade B-cell lymphomas and reactive hyperplasia. Am J Pathol. 1994;145:86-96.

20. de Boer CJ, Schuuring E, Dreef E, Peters G, Bartek J, Kluin PM, et al. Cyclin D1 protein analysis in the diagnosis of mantle cell lymphoma. Blood. 1995;86:2715-23.

21. Velders GA, Kluin-Nelemans JC, de Boer CJ, Hermans J, Noordijk EM, Schuuring E, et al. Mantle-cell lymphoma: a population-based clinical study. J Clin Oncol. 1996;14:1269-74.

22. Palaniswamy C, Selvaraj DR, Chugh T, Dudha M, Kumar U, Guleria R, et al. Mantle cell lymphoma presenting as Mikulicz syndrome. Am J Ther. 2009;16:459-61.

23. Krishnamurthy A, Shah A, Ganesan P, Sagar TG. Mantle cell lymphoma presenting as Mikulicz syndrome. J Cancer Res Ther. 2011;7:372-5.

24. Moriyama M, Furukawa S, Kawano S, Goto Y, Kiyoshima T, Tanaka A, et al. The diagnostic utility of biopsies from the submandibular and labial salivary glands in IgG4-related dacryoadenitis and sialoadenitis, so-called Mikulicz's disease. Int J Oral Maxillofac Surg. 2014;43:1276-81.

\section{Submit your next manuscript to BioMed Central and take full advantage of:}

- Convenient online submission

- Thorough peer review

- No space constraints or color figure charges

- Immediate publication on acceptance

- Inclusion in PubMed, CAS, Scopus and Google Scholar

- Research which is freely available for redistribution 